\title{
Variabilité de la sensibilité au fénarimol de souches de Venturia inaequalis provenant de vergers français
}

\author{
L Parisi ${ }^{1 *}, \mathrm{~J}$ Guillaumès 1, G Wuster 2 \\ 1 INRA, station de pathologie végétale, rue Georges-Morel, BP 57, F49071 Beaucouzé cedex; \\ ${ }^{2}$ SRPV Pays de Loire, 10, rue Le-Nôtre, F49044 Angers, France
}

(Reçu le 25 février 1994 ; accepté le 27 juillet 1994)

\begin{abstract}
Résumé - La sensibilité au fénarimol de 150 souches de Venturia inaequalis provenant de 5 vergers français a été testée par l'étude de leur croissance mycélienne sur milieu gélosé additionné de doses croissantes du fongicide. Au cours des 5 années précédant l'isolement des souches, ces vergers avaient reçu un nombre de traitements variable de 0 à 50 avec des fongicides inhibiteurs de la C-14 déméthylation (DMI). Les résultats de cette étude montrent une grande variabilité des valeurs des concentrations inhibant $50 \%$ et $90 \%$ de la croissance mycélienne $(\mathrm{Cl} 50$ et $\mathrm{Cl} 90)$ des différentes souches testées. La sensibilité des souches de chaque verger est en relation avec le nombre de traitements à base de fongicides DMI. Le verger de référence, non traité, a une population sensible significativement différente de celles des autres vergers, avec une moyenne des $\mathrm{Cl} 50$ de ses souches égale à $0,14 \mathrm{mg} / /$ de fénarimol. Les vergers traités ont des moyennes des $\mathrm{Cl} 50$ de leurs souches variant de 0,18 à 2,63 mg/l. Leurs populations varient de majoritairement sensible (cas d'un verger ayant reçu 18 traitements) à totalement résistante au fénarimol (cas d'un verger ayant reçu 50 traitements). Les différences de sensibilité de populations de $V$ inaequalis de ces vergers sont discutées en relation avec l'incidence de la maladie l'année du prélèvement et le programme de traitements fongicide de chaque verger. Les implications de ces résultats dans la stratégie d'utilisation des fongicides DMI dans la lutte anti-tavelure sont discutées.
\end{abstract}

tavelure / pommier / lutte chimique / résistance aux fongicides / fénarimol

Summary - Sensitivity to fenarimol of Venturia inaequalis strains from French orchards. The sensitivity to fenarimol of 150 Venturia inaequalis strains from 5 French orchards was tested by an agar plug assay with increasing rates of fungicide. The 5 orchards had received 0 to 50 treatments with sterol demethylation inhibitor fungicides (DMI) during the 5 years preceding the sampling. The results showed a great variability of the concentrations inhibiting $50 \%$ and $90 \%$ of the mycelial growth (ED 50 and ED 90) of the strains. The sensitivity of the strains from each orchard was related to the number of treatments with DMI fungicides. The non-treated reference orchard had a sensitive population, significantly different from the others; the mean ED 50 value of its strains was $0.14 \mathrm{mg} / \mathrm{l}$ of fenarimol. The treated orchards had mean ED 50 values ranging from 0.18 to $2.63 \mathrm{mg} /$. Their populations varied from nearly sensitive (for an orchard which had received 18 treatments) to entirely resistant to fenarimol (for an orchard which had received 50 treatments). The different sensitivities of the $\mathrm{V}$ inaequalis populations of these orchards are discussed in relation to the disease incidence on the year of sampling and the fungicide schedule. The consequences of these results on the strategy for the use of DMI fungicides against apple scab are discussed.

apple scab / apple tree / chemical control / fungicide resistance / fenarimol

\footnotetext{
* Correspondance et tirés à part
} 


\section{INTRODUCTION}

La tavelure du pommier est actuellement la principale maladie fongique des vergers français. La lutte. contre Venturia inaequalis (Cke) Wint nécessite de nombreux traitements fongicide (6 à 20 suivant les années et les régions), la majorité des variétés cultivées étant sensibles à la maladie. Ces interventions répétées posent plusieurs types de problèmes. La présence éventuelle de résidus dans les fruits à la récolte, ainsi que les effets secondaires de certains fongicides vis-àvis des populations d'auxiliaires utiles à la régulation des populations d'acariens phytophages en font partie. D'autre part, le risque de voir apparaître des souches de $V$ inaequalis résistantes à un fongicide intensivement employé ne doit pas être sous-estimé.

De ce fait, il devient indispensable de raisonner les traitements pour aboutir à une efficacité maximale tout en limitant l'émergence de ces problèmes. Cette nécessité permet d'amorcer une évolution de la lutte contre $V$ inaequalis prenant davantage en compte les particularités de chaque verger (au niveau de la sensibilité variétale et de l'inoculum présent) et les périodes de risque de contamination, grâce aux réseaux d'avertissement.

Dans ce contexte, les fongicides inhibiteurs de $\mathrm{C}$-14 déméthylation (DMI) permettent d'intervenir curativement jusqu'à $120 \mathrm{~h}$ après la pluie contaminatrice. Ils ont aussi une action préventive dont la durée est de 3 à $4 \mathrm{j}$. Leur rôle est important dans la lutte contre la tavelure, car ce sont les seuls fongicides curatifs dont on dispose actuellement. Ils permettent une intervention lorsque un risque n'est pas couvert par un traitement préventif, et l'application de stratégies de lutte raisonnée en fonction d'un seuil d'intervention (Parisi et Lefeuvre, 1992). Douze matières actives sont actuellement autorisées seules ou en mélange avec un fongicide multisite de contact, qui permet d'allonger la durée d'action préventive des DMI. Ce sont le bitertanol, le cyproconazole, le difénoconazole, le fénarimol, le fluquinconazole, le flusilazole, i'hexaconazole, le myclobutanil, le nuarimol, le penconazole, le pyrifénox et la triforine.

En 1985, Stanis et Jones ont mis en évidence pour la première fois l'existence de souches de $V$ inaequalis résistantes aux DMI dans un verger allemand. Depuis, des souches résistantes ont été isolées en France (Thind et al, 1986), en Italie (Fiaccadori et al, 1987), en Belgique (Creemers et al, 1988) et en Autriche (Hermann et al, 1989). Les facteurs de résistance (FR) de toutes ces souches ont une valeur comprise entre 4 et 20.

En 1988, au Canada, Hildebrand et al signalent pour la première fois une perte d'efficacité de ces fongicides dans un verger expérimental soumis depuis 10 ans à des traitements intensifs. Depuis, la perte pratique d'efficacité des traitements avec les DMI a été constatée dans les vergers européens, notamment en Hongrie (Enisz, 1988), en Italie (Fiaccadori et Cesari, 1989) et en Autriche (Hermann et al, 1989).

En France, les Services de la protection des végétaux ont détecté des souches résistantes dans près de la moitié des 39 échantillons analysés en 1989. L'année suivante, quelques vergers intensivement traités subissaient d'importantes attaques de tavelure; les souches issues de ces vergers étaient très majoritairement résistantes au fénarimol (Parisi et al, 1991).

Dans ce contexte, l'objectif de ce travail est d'analyser la sensibilité aux DMI de populations de $V$ inaequalis issues de vergers plus ou moins intensivement traités, afin de détecter toute modification de cette sensibilité entraînée par la pression de sélection des traitements fongicide. L'analyse de la sensibilité de populations de $V$ inaequalis issues de vergers subissant des échecs dans la lutte contre la maladie permettra éventuellement de confirmer l'hypothèse d'une perte pratique d'efficacité des DMI dans les vergers français.

\section{MATÉRIEL ET MÉTHODES}

\section{Souches de $\mathrm{V}$ inaequalis}

Les souches testées proviennent de 5 vergers français ayant des programmes de traitement contre la tavelure différents. Quatre de ces vergers sont des vergers commerciaux, avec des programmes incluant tous des fongicides DMI (tableau I). Le verger 4 est le plus intensivement traité, avec 50 applications avec le flusilazole en 3 ans ; l'année du prélèvement des échantillons, il a reçu 17 traitements et subi une forte attaque sur fruits. Le verger 5 totalise lui aussi 50 traitements avec le flusilazole mais sur 5 ans, avec environ 10 traitements par an ; 80 à $90 \%$ des fruits sont tavelés l'année du prélèvement. Le verger 3 est moins intensivement traité, car sur 5 ans 36 DMI (fénarimol et flusilazole) ont été appliqués ; l'année du prélèvement 9 traitements sont comptabilisés et $30 \%$ de fruits sont tavelés à la récolte. Le verger 2 est moins intensivement traité avec le penconazole ( 6 par an entre 1987 et 1989, 2 en 1990). Le verger 1 est le seul ver- 
Tableau I. Caractéristiques des vergers étudiés.

\begin{tabular}{|c|c|c|c|c|c|c|}
\hline Verger & $r$ Localisation & Variété & $\begin{array}{l}\text { Nb d'applications } \\
\text { de DMIl'année } \\
\text { du prélèvement a }\end{array}$ & $\begin{array}{c}\text { Cumul des } \\
\text { applications de } \\
\text { DMI depuis } \\
\text { l'année indiquée }\end{array}$ & $\begin{array}{c}\text { Principaux DMI } \\
\text { employés (seuls } \\
\text { ou en association) }\end{array}$ & $\begin{array}{l}\text { Incidence de la maladie } \\
\text { lors du prélèvement } \\
\text { (\% de fruits tavelés) }\end{array}$ \\
\hline 1 & Maine-et-Loire & Golden Delicious & 0 & $1980: 0$ & & Inconnue \\
\hline 2 & Tarn-et-Garonne & Smoothee & 2 & $1987: 18$ & Penconazole & Inconnue \\
\hline 3 & Tarn-et-Garonne & Granny Smith & 9 & $1986: 36$ & $\begin{array}{l}\text { Fénarimol, } \\
\text { Flusilazole }\end{array}$ & 30 \\
\hline 4 & Drôme & Granny Smith & 17 & $1988: 50$ & Flusilazole & 80 à 90 \\
\hline 5 & Corrèze & Lysgolden & 10 & $1986: 50$ & Flusilazole & 80 à 90 \\
\hline
\end{tabular}

a Prélèvement en octobre 1991 (verger 1) et en septembre 1990 (vergers 2 à 5).

ger non commercial inclus dans cette expérience. C'est un verger expérimental de I'INRA d'Angers ; aucun traitement n'y a été appliqué depuis sa plantation.

Les isolements sont réalisés à partir des échantillons reçus par le Groupement régional d'intérêt scientifique phytosanitaire (GRISP) d'Angers pour la détection de souches résistantes aux DMI. Le protocole de prélèvement des échantillons prévoit de collecter 50 feuilles ou fruits tavelés dans différents points de la parcelle.

Toutes les souches sont issues d'isolements monoconidiens ; leur effectif varie de 18 à 40 par verger (tableau II).

\section{Croissance mycélienne sur milieu avec fongicide}

Les essais ont été conduits avec le fénarimol. Ce fongicide était le DMI le plus employé dans les vergers de pommiers lors des premiers travaux de détection entrepris par le GRISP de Bordeaux (Morzières et al, 1988 ) ; de ce fait il a été choisi comme fongicide de référence pour les tests de détection. Dans le cas de $V$ inaequalis, la résistance paraît croisée positive entre
DMI (Thind et al, 1986). La bonne corrélation entre résultats obtenus avec le fénarimol et résultats obtenus avec d'autres DMI apparus plus récemment dans la lutte anti-tavelure a par ailleurs été vérifiée (Parisi, Wuster, résultats non publiés).

Le fongicide (spécialité commerciale: Rubigan 4) est ajouté à du malt gélosé (Cristomalt de Difal à 1\%) maintenu en surfusion. Dix concentrations de matière active ont été testées : $0 ; 0,05 ; 0,1 ; 0,25 ; 0,5 ; 1$; 2,$5 ; 5 ; 7,5$ et $10 \mathrm{mg} / \mathrm{l}$. Par souche et par concentration, 3 boîtes de Petri sont ensemencées avec 4 implants de $4 \mathrm{~mm}$ de diamètre pris à la périphérie d'une colonie de $V$ inaequalis âgée d'environ 1 mois. L'incubation dure un mois à l'obscurité et à une température de $16 \pm 1{ }^{\circ} \mathrm{C}$, puis le diamètre de chaque colonie est mesuré.

Le pourcentage d'inhibition de la croissance mycélienne en présence de fongicide est calculé comparativement aux témoins sans fongicide, puis les concentrations inhibant 50 et $90 \%$ de la croissance mycélienne ( $\mathrm{Cl} 50$ et $\mathrm{Cl} 90)$ sont estimées graphiquement.

En fonction des valeurs des $\mathrm{Cl} 50$ transformées en logarithmes, 8 classes de souches de $V$ inaequalis ont été définies, d'étendue 0,3 avec $-0,9$ comme borne inférieure. Avec les données non transformées, les bornes de chaque classe sont définies dans le tableau III.

Tableau II. Variabilité des concentrations de fénarimol inhibant 50 et $90 \%$ de la croissance mycélienne de 150 souches de $V$ inaequalis provenant des 5 vergers.

Verger Nombre de souches testées
Amplitude des valeurs des C150 ( $m g /$ fénarimol)
Amplitude des valeurs des $\mathrm{Cl} 90$ ( $m g / l$ fénarimol)

$\begin{array}{lllr}1 & 37 & <0,05 \text { à } 0,52 & <0,05 \text { à } 0,90 \\ 2 & 32 & 0,05 \text { à } 0,60 & 0,25 \text { à } 2,02 \\ 3 & 23 & 0,08 \text { à } 4,95 & 0,45 \text { à } 7,45 \\ 4 & 40 & 0,57 \text { à } 5,5 & 2,10 \text { à }>10 \\ 5 & 18 & 0,40 \text { à } 8,05 & 1,80 \text { à }>10\end{array}$


Tableau III. Classes de sensibilité au fénarimol.

\section{Classe Valeurs des $\mathrm{Cl} 50 \mathrm{en} \mathrm{mg} / \mathrm{l}$ de fénarimol}

\begin{tabular}{cc}
\hline & \\
$\mathrm{a}$ & $\mathrm{Cl} 50 \leq 0,125$ \\
$\mathrm{~b}$ & $0,125<\mathrm{Cl} 50 \leq 0,25$ \\
$\mathrm{c}$ & $0,25<\mathrm{Cl} 50 \leq 0,5$ \\
$\mathrm{~d}$ & $0,5<\mathrm{Cl} 50 \leq 1$ \\
$\mathrm{e}$ & $1<\mathrm{Cl} 50 \leq 2$ \\
$\mathrm{f}$ & $2<\mathrm{Cl} 50 \leq 4$ \\
$\mathrm{~g}$ & $4<\mathrm{Cl} 50 \leq 8$ \\
$\mathrm{~h}$ & $\mathrm{Cl} 50>8$
\end{tabular}

Les souches appartenant aux classes a, b et c sont considérées comme sensibles, les souches des classes d à $h$ comme ayant une sensibilité réduite au fénarimol.

\section{Analyse statistique}

La sensibilité aux DMI de la population des différents vergers a été comparée à l'aide du test non paramétrique $U$ de Mann et Withney, la condition d'homogénéité des variances, nécessaire pour réaliser une analyse de variance, n'étant pas remplie (tableau IV) (test de Bartlett : probabilité associée inférieure à 0,001). Le test a été réalisé avec les valeurs des $\mathrm{Cl} 50$ des souches de chaque verger.

\section{RÉSULTATS}

Une grande variabilité des valeurs des $\mathrm{Cl} 50$ et $\mathrm{Cl} 90$ est observée parmi les 150 souches testées (tableau II). Les CI 50 varient en effet d'inférieures à $0,05 \mathrm{mg} / \mathrm{l}$ de fénarimol à $8,05 \mathrm{mg} / \mathrm{l}$, les $\mathrm{Cl} 90$ d'inférieures à $0,05 \mathrm{mg} / \mathrm{l}$ à supérieures à $10 \mathrm{mg} / \mathrm{l}$. Cette variabilité est fonction du verger d'origine des souches. Les souches issues des vergers 1 et 2 ont des $\mathrm{Cl} 50$ comprises entre inférieures à $0,05 \mathrm{mg} / \mathrm{l}$ et $0,6 \mathrm{mg} / \mathrm{l}$, et des $\mathrm{Cl} 90$ variant d'inférieures à $0,05 \mathrm{mg} / \mathrm{l}$ à $2,02 \mathrm{mg} / \mathrm{l}$. Les $\mathrm{Cl} 50$ des souches issues des vergers 3,4 et 5 sont comprises entre 0,08 et $8,05 \mathrm{mg} / \mathrm{l}$, tandis que leurs $\mathrm{Cl} 90$ varient de 0,45 à supérieures à $10 \mathrm{mg} / \mathrm{l}$. La moyenne des $\mathrm{Cl} 50$ des souches isolées de chaque verger varie de $0,14 \mathrm{mg} / \mathrm{l}$ pour le verger 1 à $2,63 \mathrm{mg} / \mathrm{l}$ pour le verger 5 (tableau IV) ; cette valeur est plus forte dans les vergers ayant reçu un plus grand nombre de DMI. On note en particulier une différence d'un facteur 11,6 à 18,8 entre la $\mathrm{Cl} 50$ moyenne des souches du verger de référence et celle des vergers 3 à 5, intensivement traités avec les DMI (tableau IV).

La structure des populations du champignon, telle qu'elle est révélée par la distribution dans les 8 classes de sensibilité montre des différences notables suivant le verger d'origine des souches (fig 1). La quasi-totalité des souches issues des vergers 1 et 2 se répartissent dans les classes $\mathrm{a}, \mathrm{b}$ et $\mathrm{c}$ avec une majorité d'entre elles en classe a (verger 1) ou b (verger 2). En revanche, pour les vergers 3,4 et 5 , les souches appartenant aux classes $a, b$ et $c$ sont minoritaires (verger 3 et 5), ou inexistantes (verger 4). Pour ces 3 vergers, la majorité ou totalité des souches se répartissent dans les classes d à $\mathrm{g}$.

Ces observations sont confortées par les résultats de la comparaison des différents vergers par le test de Mann et Withney (tableau IV). Les populations des vergers 2 à 5 sont significativement moins sensibles aux DMI que celle du verger de référence 1 ; les populations des vergers 4 et 5 sont significativement moins sensibles que celles des vergers 2 et 3 .

Tableau IV. Comparaison de la sensibilité au fénarimol des populations de $V$ inaequalis provenant des 5 vergers.

\begin{tabular}{ccccc}
\hline Verger & $\begin{array}{c}\text { Moyenne des Cl } 50 \\
\text { des souches testées }\end{array}$ & Variance & $\begin{array}{c}\text { Facteur de } \\
\text { résistance a }\end{array}$ & $\begin{array}{c}\text { Comparaison de la sensibilité des vergers } \\
\text { par le test U de Mann et Withney }\end{array}$ \\
\hline & & $7,5610^{-4}$ & - & $\mathrm{a}$ \\
2 & 0,14 & $1,3410^{-2}$ & 1,28 & $\mathrm{~b}$ \\
3 & 0,18 & 2,04 & 11,6 & $\mathrm{c}$ \\
4 & 1,62 & 0,77 & 13,4 & $\mathrm{~d}$ \\
5 & 1,88 & 4,24 & 18,8 & $\mathrm{~d}$ \\
\hline
\end{tabular}

a Moyenne des Cl 50 des populations des vergers 2, 3, 4 ou 5/moyenne des Cl 50 de la population du verger 1. 

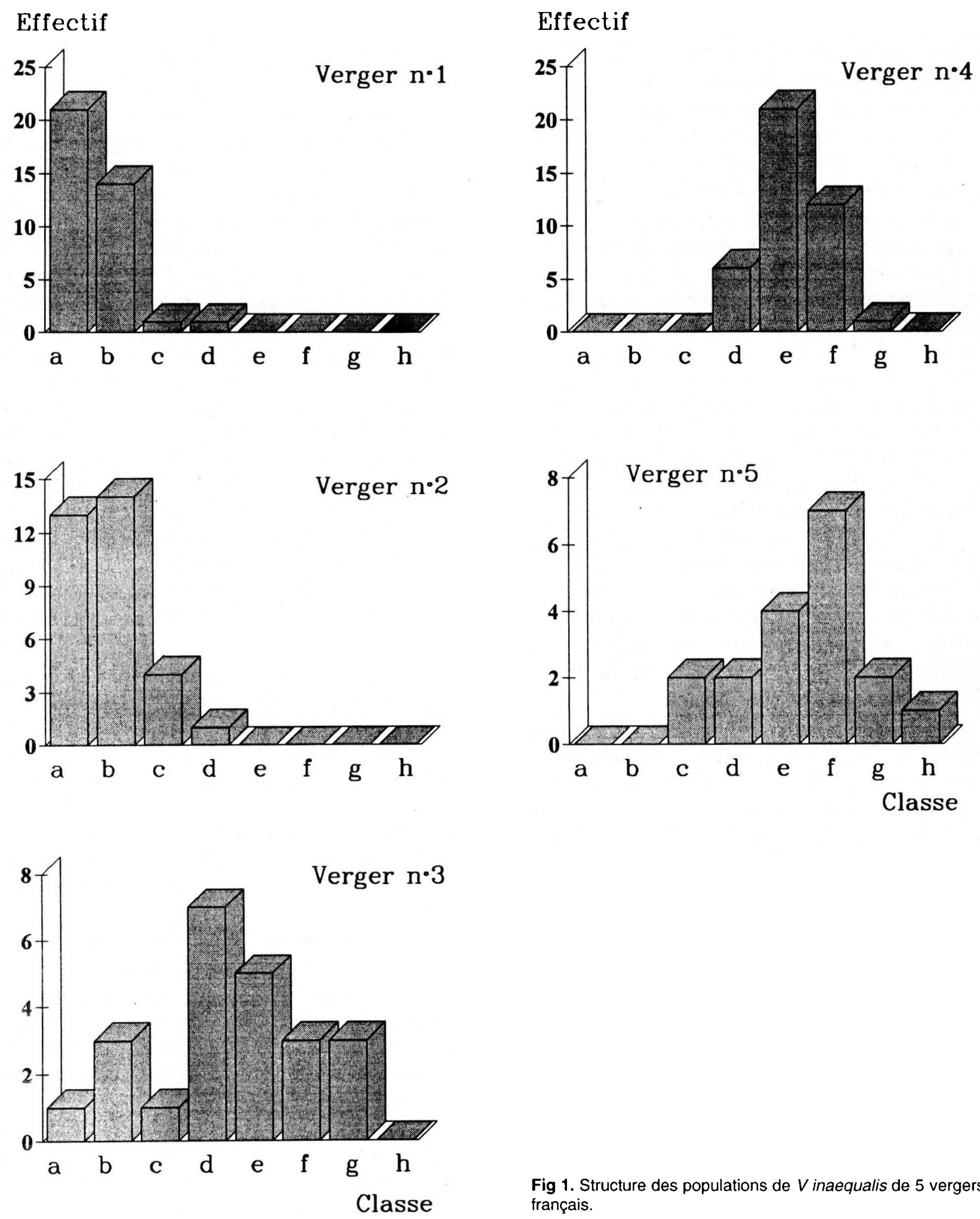

Fig 1. Structure des populations de $V$ inaequalis de 5 vergers français.

\section{DISCUSSION}

La sensibilité au fénarimol des souches de $V$ inaequalis testées au cours de cette étude paraît être clairement en relation avec le nombre de traitements avec les DMI appliqués dans leur verger d'origine.

Ces résultats montrent, pour la première fois en France, dans des vergers commerciaux, un déplacement significatif de la sensibilité des populations de $V$ inaequalis après des traitements intensifs avec les fongicides DMI. Ils sont en accord avec ceux de Braun et McRae (1992) qui, au Canada, mettent en évidence une sous- 
population résistante au myclobutanil dans un verger intensivement traité. Dans ce cas, le facteur de résistance (moyenne des $\mathrm{Cl} 50$ de la population résistante/moyenne des $\mathrm{Cl} 50$ de la population sensible) est de 10,2. Dans nos vergers, avec le fénarimol, les facteurs de résistance sont compris entre 11,6 et 18,8 .

Les vergers 3 à 5 de notre essai, dans lesquels des populations de $V$ inaequalis totalement ou partiellement résistantes au fénarimol sont mises en évidence, subissent tous les 3 des pertes dues à la maladie, malgré des calendriers de traitements parfois très chargés (jusqu'à 17 DMI pour le verger 4). Bien que nous ne puissions en apporter la preuve formelle, faute de données vérifiables sur les conditions d'application des fongicides (dosage réel, pulvérisation, lessivage), nous pensons qu'il s'agit des premiers cas de résistance en pratique aux DMI enregistrés en France. Nos résultats concordent donc avec ceux de Hildebrand et al (1988) qui mettent en évidence une perte pratique d'efficacité par paliers. Le verger 3 , qui subit $30 \%$ de perte, a une population moins résistante que les vergers 4 et 5 , où la perte d'efficacité paraît totale (80 à $90 \%$ de fruits tavelés).

Aux États-Unis, en revanche, Smith et al (1991) ne trouvent pas de différence significative entre la sensibilité au flusilazole d'une population de $V$ inaequalis provenant d'un verger traité depuis 12 ans et celle de 2 vergers non traités. Le verger traité n'était cependant pas un verger commercial, et avait subi des programmes de traitements complexes incluant des fongicides autres que les DMI. Dans notre étude, le programme de traitement des vergers 3 à 5 était presque exclusivement basé sur l'utilisation des fongicides DMI, seuls (vergers 3 et 4) ou associés à un fongicide multisite de contact (verger 5).

La perte d'efficacité par paliers observée dans cette étude, ainsi que la distribution dans les 8 classes de sensibilité des souches provenant des 5 vergers évoquent le développement d'une résistance de type polygénique. Dans ce cas, la population initiale a une distribution unimodale, comme pour le verger 1 de notre étude, non traité avec les DMI ; la pression de sélection exercée par les traitements fongicide provoque une diminution graduelle de la sensibilité des populations (Wolfe, 1982 ; Skylakakis, 1985). Dans notre cas, cette évolution progressive vers les moindres sensibilités s'observe pour les vergers 2 à 5 , en fonction du nombre de traitements fongicide reçu par chaque verger. Le déterminisme polygénique de la résistance aux DMI a été mis en évidence chez d'autres champignons, dont Erisiphe graminis $f \mathrm{sp}$ hordei (Leroux, 1991).

Le programme de traitement des vergers concernés par cette étude pose le problème des stratégies à mettre en œuvre pour éviter ces situations de résistance en pratique. Dès 1987, I'INRA et les Services de la protection des végétaux mettaient en garde les arboriculteurs contre une utilisation systématique et intensive des fongicides DMI, à la suite des premières détections de souches résistantes en Europe (Thind et al, 1986). Bien que ces fongicides aient été classés à risque faible à modéré vis-à-vis de la résistance (Dekker, 1985 ; Georgopoulos et Skylakakis, 1986), un certain nombre de données concernant les oïdiums des céréales et des cucurbitacées et le piétin-verse des céréales mettaient en évidence des pertes d'efficacité au champ (Leroux, 1987). Dans la pratique, 3 à 4 applications avec les DMI par an au maximum, réservées aux situations où une contamination n'avait pas pu être couverte par un traitement préventif, étaient préconisées.

Depuis, la résistance aux fongicides DMI a progressé et touche un plus grand nombre de champignons pathogènes (Leroux, 1991). Chez les plantes pérennes, Uncinula necator, agent de l'oïdium de la vigne est aussi concerné par ce problème. Des souches d'U necator résistantes aux DMI sont fréquemment détectées dans les vignobles européens, bien que l'efficacité des DMI reste dans l'ensemble satisfaisante (Steva, 1994).

En ce qui concerne la tavelure du pommier, il est évident que le type de lutte préconisé dès 1987 demande la prise en compte des informations fournies par les réseaux d'avertissement, et une plus grande souplesse d'intervention quand un traitement stop ou curatif est nécessaire. C'est la raison pour laquelle elle n'est pas employée par tous les producteurs. C'est cependant une stratégie qui se révèle efficace, car les arboriculteurs pratiquant cette lutte raisonnée n'ont pas rencontré à ce jour de problème pouvant être relié à une situation de résistance. Cependant, nous manquons de données plus précises concernant la sensibilité des populations de $V$ inaequalis issues de ce type de verger, traité 3 à 4 fois par an depuis une dizaine d'années.

Le verger 2 de cette étude est un cas intéressant. Après une analyse détectant $9 \%$ de conidies résistantes au fénarimol en 1989 , le rythme de 5 à 6 traitements avec les DMl est réduit à 2 
par an en 1990. Les résultats de notre étude montrent que, bien que la moyenne des $\mathrm{Cl} 50$ de ce verger soit supérieure à celle de notre verger de référence, la grande majorité des souches issues de ce verger en 1990 peuvent être considérées comme sensibles (classes a à $\mathrm{c}$ ). En 1991, 2 traitements avec les DMI sont réalisés dans ce verger et une nouvelle analyse détecte $3 \%$ de conidies résistantes (Wuster, résultats non publiés).

En ce qui concerne une des stratégies antirésistance préconisées par Scheinpflug (1988), à savoir l'association d'un fongicide multisite de contact avec les DMI, il est intéressant de remarquer que les vergers 4 et 5 de notre étude étaient traités avec le même DMI, le flusilazole, seul (verger 4) ou associé à un fongicide de contact à demi-dose (verger 5). Cette association n'évite donc pas l'apparition de souches résistantes. Nous n'avons aucun élément pour savoir s'il en aurait été de même avec une pleine dose de produit de contact, ni si l'association a contribué à ralentir le phénomène.

Nos résultats montrent clairement le danger de l'utilisation intensive des fongicides DMI dans la lutte contre la tavelure du pommier. Ce sont les seuls qui permettent, pour l'instant, une intervention curative. Pour préserver leur efficacité, il faut impérativement les utiliser à bon escient. Les vergers en situation de résistance risquent de rencontrer de graves difficultés dans la lutte contre la maladie. Ils présentent souvent un inoculum fort à l'automne, sans qu'il existe de réelle possibilité de le réduire. Si les conditions climatiques du printemps suivant sont favorables à la maladie, la lutte avec les seuls fongicides de contact peut être extrêmement difficile à conduire dans ces vergers.

\section{REMERCIEMENTS}

Les auteurs remercient $J$ Gaudin pour sa collaboration technique et $\mathrm{J}$ Luisetti pour ses conseils en statistique.

\section{RÉFÉRENCES}

Braun PG, McRae KB (1992) Composition of a population of Venturia inaequalis resistant to myclobutanil. Can J Plant Pathol 14, 215-220

Creemers P, Vandergeten J, Vanmechelen A (1988) Variability in sensitivity of fields isolates of Venturia $\mathrm{sp}$ to demethylation inhibitors. Meded Fac Landbouwwet Rijksuniv Gent 53/2b, 577-587
Dekker J (1985) The development of resistance to fungicides. Prog Pestic Biochem Toxicol 4, 165-218

Enisz $\mathrm{J}$ (1988) A method for measurement in vivo of the sensitivity to SBI-fungicides of Venturia inaequalis. ISPP Chemical Control Newsletter 11, 36-37

Fiaccadori R, Gienlink AJ, Dekker J (1987) Sensitivity to inhibitors of sterol biosynthesis in isolates of Venturia inaequalis from Italian and Deutch orchards. Neth J Plant Patho/ 93, 285-287

Fiaccadori R, Cesari A (1989) Modificazioni della sensibilità di Venturia inaequalis in Italia nei confronti dei fungicidi IBE. Inf Fitopatol 12, 56-58

Georgopoulos SG, Skylakakis G (1986) Genetic variability in the fungi and the problem of fungicide resistance. Crop Prot 5, 299-305

Hermann M, Szith R, Zinkernagel V (1989) Reduced sensitivity of some scab isolates (Venturia inaequalis) from Steiermark (Austria) to EBI-fungicides. Gartenbauwissenschaft 54, 160-165

Hildebrand PD, Lockhart CL, Newbery RJ, Ross RG (1988) Resistance of Venturia inaequalis to bitertanol and other demethylation-inhibiting fungicides. Can J Plant Pathol 10, 311-316

Leroux $P$ (1987) La résistance des champignons aux fongicides-1I. Phytoma 386, 31-35

Leroux $P$ (1991) Résistance des champignons phytopathogènes aux fongicides. Phytoma 434, 20-26

Morzières JP, Clerjeau M, Thind TS (1988) Mise au point sur la résistance des souches de Venturia inaequalis aux fongicides inhibiteurs de la biosynthèse de l'ergostérol en France. Maladies des plantes, journées d'études ANPP 2, 1321-1326

Parisi L, Lefeuvre M (1992) Une approche de la lutte raisonnée contre la tavelure du pommier : choix d'une courbe de Mills comme seuil de traitement. Adalia 22-23-24, 58-59

Parisi L, Guillaumès J, Wuster G (1991) Résistance de Venturia inaequalis aux fongicides inhibiteurs de la biosynthèse des stérols : détection et caractérisation des souches en 1989 et 1990. Maladies des Plantes, journées d'étude ANPP 2, 853-862

Scheinpflug $H$ (1988) Resistance management strategies for using DMI fungicides. In : Fungicide Resistance in North America (CJ Delp, ed), Amer Phytopathol Soc, Saint Paul, MN, États-Unis, 93-94

Skylakakis G (1985) Two different processes for the selection of fungicide-resistant sub-populations. Bull OEPP 15, 519-525

Smith FD, Parker DM, Köller W (1991) Sensitivity distribution of Venturia inaequalis to the sterol demethylation inhibitor fluzilazole: baseline sensitivity and implications for resistance monitoring. Phytopathology 81, 392-396

Stanis VF, Jones AL (1985) Reduced sensitivity to sterol inhibiting fungicides in field isolates of Venturia inaequalis. Phytopathology 75, 1098-1101

Steva H (1994) Évolution de la sensibilité des populations d'oïdium aux DMI après quatre années de traitements. Phytoma 461, 31-34 
Thind T, Clerjeau M, Olivier JM (1986) First observations on resistance in Venturia inaequalis and Guinardia bidwellii to ergosterol biosynthesis inhibitors in France. Proc British Crop Prot Conf Pests Dis 2, 491-498
Wolfe MS (1982) Dynamics of the pathogen population caused by resistance to fungicides. In : Fungicide Resistance in Crop Protection (Dekker J, Georgopoulos SG, eds), Pudoc, Wageningen, Pays-Bas, 139-148 